\title{
ARCHITECTURE FOR ENVIRONMENTAL DATA ACCESS IN WSN
}

\author{
J. M. Mora-Merchan, F. J. Molina, D. F. Larios, G. Rodriguez, J. Barbancho and C. León \\ Department of Electronic Technology, University of Seville, University School Polytechnics, Seville, Spain \\ \{jmmora,fjmolina\}@us.es, dflarios@dte.us.es, \{gusrodri,jbarbancho, cleon\}@us.es
}

Keywords: $\quad$ WSN, Software Architecture, Hardware Architecture, Weather Monitoring.

\begin{abstract}
This paper shows different issues found in the real implementation of either a WSN or its data exploitation system for an environmental monitoring application. A generic software architecture for interfacing both is proposed and tested on a real case.
\end{abstract}

\section{PROBLEMS OF INTERFACING WITH WSNS}

Most papers about Wireless Sensor Networks (WSNs) do not include information about accessing data from external applications.

Usually, articles present the interface as a "sink" without mentioning its internal architecture or how it provides information to remote machines, applications or users. Figure 1 shows one of these schemes (taken from (Akyildiz et al., 2002)). Generally, scientific research is focused on WSN topology, mesh types or node characteristics, but there is a lack of information about the data flow between the the Base Station and the information system.

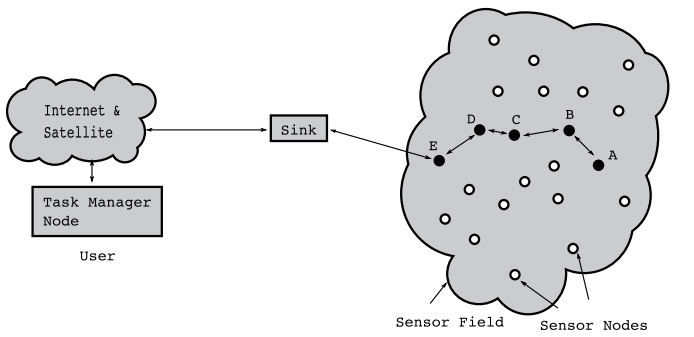

Figure 1: Typical architecture description (copy from (Akyildiz et al., 2002)).

The development and further implementation of a real WSN and an Information System require answering the following questions:

- How to connect the Sensor Field to the Sink.

- How to pre-process to validate information received, and

- How to store retrieved information.
Firstly, one involves the router needed to interconnect WSN and more practical networks like Internet. For this, it is necessary to define hardware and software communication protocols. The second question defines how to detect invalid data and how to fix it, if possible. Unlike other networks, WSNs routings are not always reliable. No packets, multiple copies of the same one, or corrupted packets arrive at the Base Station. Finally, the third question looks for an efficient data storage to allow us to keep the needed data and an easy way of retrieving information. The designing and the implementation of a WSN require finding a solution for all these questions.

The following section (sec. 2) describes the hardware of the network we are working with. In section 3 , a generic software architecture is proposed, and a real implementation is described. Finally, we summarize the main conclusions and future work.

\section{ICARO NETWORK}

On April 8th, 2010 a WSN was deployed in Doñana Biological Reserve (DBR). Doñana Biological Reserve is considered one of the most important natural protected landscapes in the world: $543 \mathrm{~km}^{2}$, of which $135 \mathrm{~km}^{2}$ are a protected area. In 1994 UNESCO designated it a World Heritage Site and the park was recognized as a Biosphere reserve. DBR is located in the southwest of Andalusia (Spain).

The network, called Icaro, was devised as a pilot of environmental measurements and distributed computation. Its purpose is to serve as a real testing ground. It allows us to check our simulations in real conditions. Icaro lets us measure interactions not considered in theoretical models. 
Each node in Icaro is a stand alone system capable of running as an independent entity or within a collaborative network. It is made up of 4 functional blocks shown in figure 2: Sensors, Power Supply, Processing Unit and Communications.

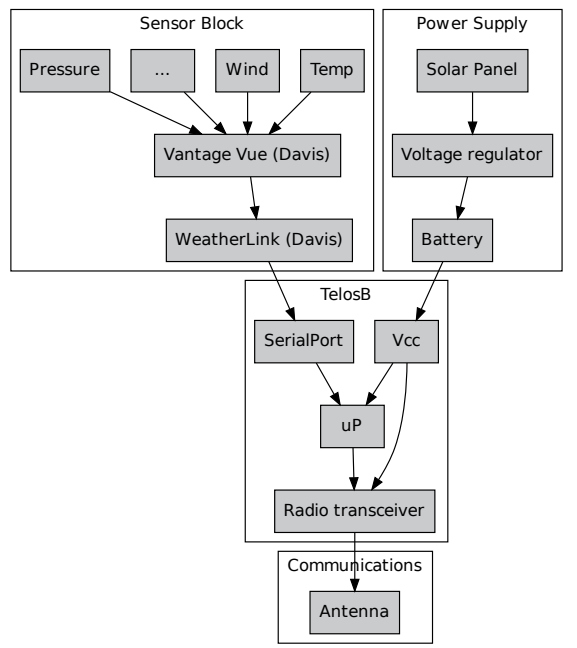

Figure 2: Node Hierarchy.

Sensor Block is the interface with the environment. Sensors are integrated in a commercial weather station (Davis Vantage Vue (Davis Instruments, nda)) which captures temperature, atmospheric pressure, humidity, rain rate, wind velocity and direction. It contains a custom module for serial communications (Davis WeatherLink 6510SER (Davis Instruments, ndb)) to transfer information to the Processing Unit. In the Power Supply block, a $12 \mathrm{~V} 6$ Ah battery stores and distributes energy harvested by a $10 \mathrm{~W}$ solar panel.

A Crossbow TelosB mote (Crossbow, nd) is the core processing unit. It is an inexpensive platform which has an integrated radio transceiver and an accessible serial port. TelosB is a platform supported by TinyOS (Levis et al., 2005) operating system, It is very popular and well tested by the research community on WSN applications. TinyOS provides a communication stack and manages access to peripherals. This simplifies the development of new algorithms.

For the communications, TelosB includes a transceiver. To increase radio range, we have added an external omnidirectional antenna.

Figure 3 shows a real installed node in Doñana. The pole holds the weather station, the solar panel, antenna, and a sealed box with the rest of the components.

Icaro topology is shown in figure 4. Ten nodes and a base station make up the Icaro network. The arrows in the figure represent the routes that packets can follow to the Base Station. The topology has been

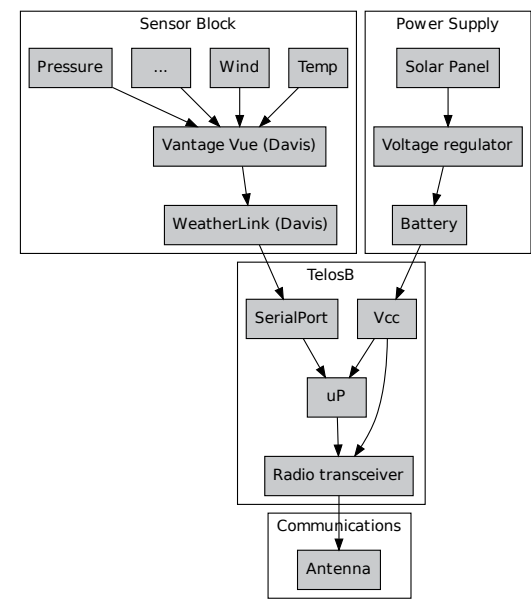

Figure 3: Real Icaro Node.

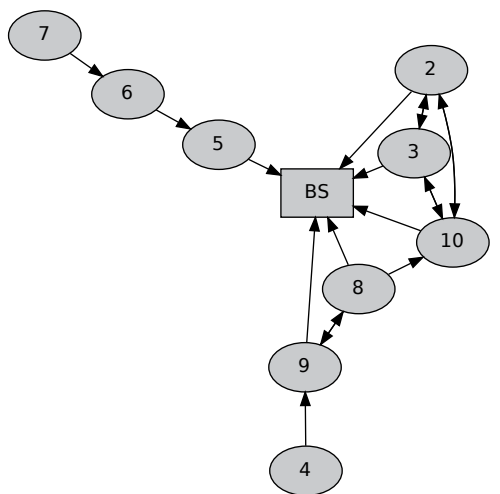

Figure 4: Icaro Topology.

designed to test different cases (Stinnett, 2007)(Patrikar and Akojwar, 2008)(Thouvenin, 2007): Pure multi-hop routes (nodes 5, 6 and 7), pure all-to-all nets (nodes 2, 3 and 10, and nodes 8 and 9 ) and hybrid combinations (nodes 4, 8, 9 and 10).

Base Station is a node with a Moxa embedded industrial PC (Moxa, nd) connected to TelosB by USB. TelosB software of Base Station collects data from network and re sends to the PC. This PC acts as a bridge between WSN and Internet.

\section{SOFTWARE ARCHITECTURE FOR THE INTERFACE BLOCK}

\subsection{General Architecture}

We propose an architecture (fig. 5) for interfacing data collected in a WSN to final applications. As mentioned, this interface is usually overlooked in scientific papers, and it should be part of a general scheme. 


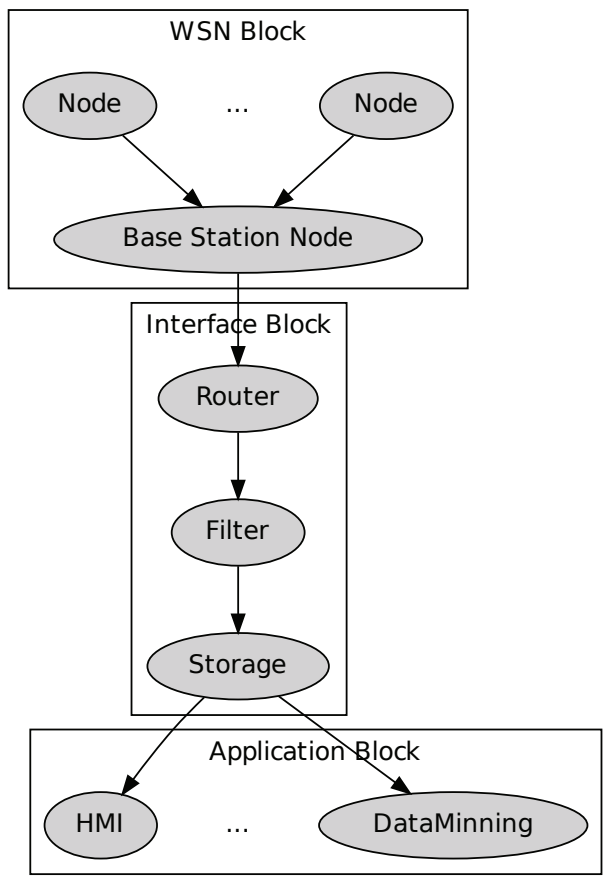

Figure 5: General Architecture.

WSN is a sensor field where nodes are data sources which provide raw data, processed data and metadata. Raw data are direct measurements of the sensors. Processed data are the result of individual or collective distributed processing of several nodes, and metadata facilitate data interpretation like timestamps, geographic information, data range, etc.

In our model, processing algorithms are considered as virtual sensors with the same logic internal structure and methods of the real sensors. In fact, they can associate metadata to the processed data.

Depending on the application, results of collaborative processing may be assigned to the area covered by the involved sensors. In these cases, virtual nodes must be created using special node identifiers, global information, such as time, and area-related parameters. With this approach, all data management from Base Station to application level is homogeneous, no matter the origin of the data.

Interface Software Architecture is made up of three software components: router, filter, and storage.

The router translates the data frames from WSN to a more suitable one, and it must open a TCP server socket. In this way, multiple clients can access the data stream, no matter if they are in the same host machine.

In order to test all wireless traffic, the router will resend all incoming communication frames, not only sensor data frames. The filter component is highly dependent on the WSN application, and it must often be customized to select the right sensor data, and to remove corrupted or duplicated data.

Storage component saves either filtered on unfiltered data to facilitate data retrieval, tracing and debugging.

\subsection{Icaro Interface Software Architecture}

Icaro implements a customized architecture of the general model (fig. 5). It consists of independent modules so that it is possible to improve the system replacing one or more modules. Figure 6 shows the real structure of Icaro.

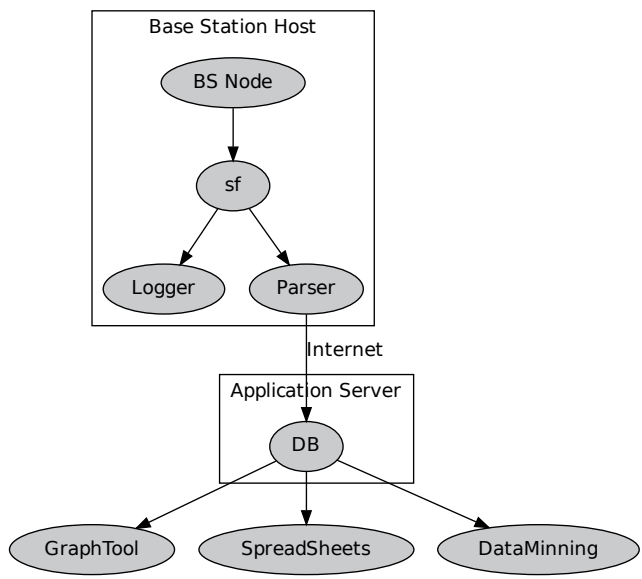

Figure 6: Software Architecture of the Interface.

The interface functionality is divided into the Base Station Computer and Application Computer. Base Station Computer is a Moxa industrial embedded PC with a TelosB mote plugged in a USB port. The second computer is a remote Database (Mysql) placed in our laboratory.

The WSN data are timestamped and geographic information is coded in the node identification. Furthermore, Icaro runs an auto-organized neural algorithm to process different environmental measurements (temperature, humidity, atmospheric pressure, etc) in el Ojillo marsh to learn predicting water level changes. The result of data processing also includes metadata with current time and debugging information (e.g. local winning neuron).

Base Station Node delivers all data to the computer host by mean of a well known TinyOS sniffer called basestation. The interface component receives all information from nodes at the entry point of the Router component. In Icaro, a TinyOS tool called Serial Forwarder (sf) implements this function.

Serial Forwarder protocol is very simple: A one byte header with the length of message and the mes- 


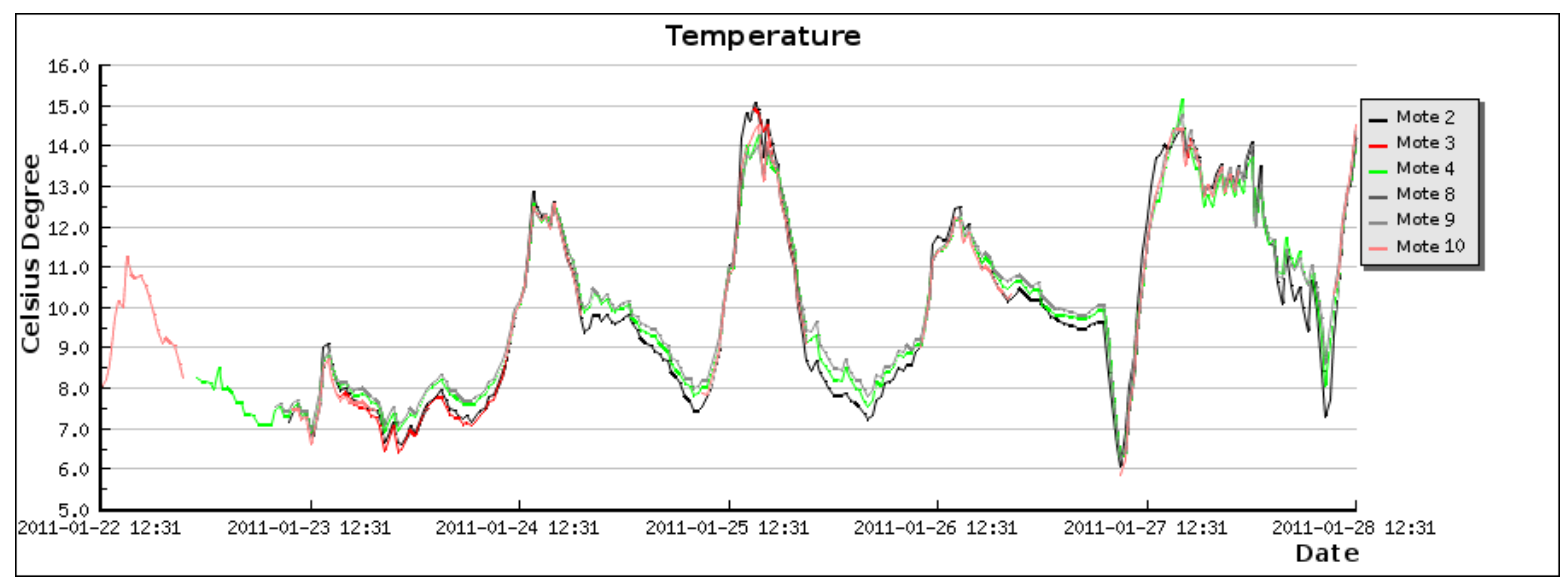

Figure 7: Example of captured data.

sage. There is no error control.

$$
\langle\text { header : length(1 byte) }\rangle\langle\text { payload : data }\rangle
$$

Data stream is sent from serial forwarder to a log registry and a filter application. The registry is a time limited copy for data frame debugging. In Icaro, the filter just removes non valid data (i.e. corrupted or duplicated) because of the lack of error control in serial forwarder protocol. A parser in the filter analyzes frames and looks for valid structures in the payload. The filter must check for multiple copies of the same message before uploading data to the DB. Table 1 shows error statistics based on a sample of 1300 cases.

Table 1: Detected errors in Parse time.

\begin{tabular}{lr} 
Valid data frame & $92.2 \%$ \\
Corrupted data frame & $3.5 \%$ \\
Duplicated data frame & $4.3 \%$ \\
\hline
\end{tabular}

The Application Server contains a database (DB) to connect with different applications to analyse data: e.g graph visualisation tools, Protocol analysers, Data Mining utilities or any other customized application. Database offers an common interface to make the data more accessible.

\section{RESULTS AND FUTURE WORK}

Designing a generic data exploitation system of a WSN is a non trivial and open problem. Most of implemented systems are application-specific.

In this paper, we present a first draft of a general software architecture to interface WSN and remote applications. A real implementation is also included to support a smart environmental application.
Icaro Net has been working since 2010. It has more than 1 GigaByte of environmental measurements. Figure 7 is a sample of temperature evolution for a week.

After analyzing the data exploiting system, we can point out the following topics to work on in the future:

- Descriptions of WSN data and metadata should be standardized, probably using a specific extension of XML.

- Virtual nodes created to represent collaborative processing results are a special sort of metadata that deserve specific attention.

- Because of specific data format in WSN applications, an automatic parser generation tool will be useful to implement new filters.

- Database time scalability. Database size is continuously growing, making data access less efficient.

\section{REFERENCES}

Akyildiz, I. F., Su, W., Sankarasubramaniam, Y., and Cayirci, E. (2002). Wireless sensor networks: a survey. Computer Networks, 38:393 - 422.

Crossbow (n.d.). Telosb datasheet. http:// www.willow.co.uk/TelosB_Datasheet.pdf. [Accessed 20 Febrary 2011].

Davis Instruments (n.d.a). Vantage Vue Weather Station. data sheet. http://www.vantagevue.com/ product_documents/weather/spec_sheets/6250_6351_ 57_SS.pdf. [Accessed 20 Febrary 2011].

Davis Instruments (n.d.b). Weather Link for Vantage Pro and Vantage Pro2 datasheet. http:// www.vantagevue.com/product_documents/weather/ spec_sheets/6510_40_44_50_60_SpecWLWin_Rev_D. pdf. [Accessed 20 Febrary 2011].

Levis, P., Madden, S., Polastre, J., Szewczyk, R., Whitehouse, K., Woo, A., Gay, D., Hill, J., Welsh, M., 
Brewer, E., and Culler, D. (2005). Tinyos: An operating system for sensor networks. In Weber, W., Rabaey, J. M., and Aarts, E., editors, Ambient Intelligence, pages 115-148. Springer Berlin Heidelberg.

Moxa (n.d.). V2101 series datasheet. http:// www.moxa.com/support/search_result.aspx?prod_id= 726\&type_id=5\&cat_type=doc. [Accessed 20 Febrary 2011].

Patrikar, R. M. and Akojwar, S. G. (2008). Neural network based classification techniques for wireless sensor network with cooperative routing. In Proceedings of the 12th WSEAS international conference on Communications, pages 433-438, Stevens Point, Wisconsin, USA. World Scientific and Engineering Academy and Society (WSEAS).

Stinnett, J. (2007). The COMPASS multihop framework for tinyos. Technical report, Rice University.

Thouvenin, R. (2007). Implementing and evaluating the dynamic manet on-demand protocol in wireless sensor networks. Master's thesis, University of Aarhus Department of Computer Science. 